Article

\title{
Comprehensive Characterization of Structural, Electrical, and Mechanical Properties of Carbon Nanotube Yarns Produced by Various Spinning Methods
}

\author{
Takayuki Watanabe ${ }^{1}$, Satoshi Yamazaki ${ }^{2}$, Satoshi Yamashita ${ }^{2}$, Takumi Inaba ${ }^{1}$, Shun Muroga ${ }^{1}$ (D), \\ Takahiro Morimoto ${ }^{1}$, Kazufumi Kobashi ${ }^{1}$ and Toshiya Okazaki ${ }^{1, * \mathbb{D}}$
}

1 CNT-Application Research Center, National Institute of Advanced Industrial Science and Technology, Tsukuba 305-8565, Japan; takayuki.watanabe.r@gmail.com (T.W.); takumi.inaba@aist.go.jp (T.I.); muroga-sh@aist.go.jp (S.M.); t-morimoto@aist.go.jp (T.M.); kobashi-kazufumi@aist.go.jp (K.K.)

2 Research Association of High-Throughput Design and Development for Advanced Functional Materials (ADMAT), Tsukuba 305-8565, Japan; satoshi.mayama.yamazaki@furukawaelectric.com (S.Y.); satoshi.yamashita@furukawaelectric.com (S.Y.)

* Correspondence: toshi.okazaki@aist.go.jp

check for updates

Citation: Watanabe, T.; Yamazaki, S.; Yamashita, S.; Inaba, T.; Muroga, S.; Morimoto, T.; Kobashi, K.; Okazaki, T. Comprehensive Characterization of Structural, Electrical, and Mechanical Properties of Carbon Nanotube Yarns Produced by Various Spinning

Methods. Nanomaterials 2022, 12, 593. https://doi.org/10.3390/nano12040593

Academic Editor: Simone Morais

Received: 12 January 2022

Accepted: 4 February 2022

Published: 10 February 2022

Publisher's Note: MDPI stays neutral with regard to jurisdictional claims in published maps and institutional affiliations.

Copyright: () 2022 by the authors. Licensee MDPI, Basel, Switzerland. This article is an open access article distributed under the terms and conditions of the Creative Commons Attribution (CC BY) license (https:// creativecommons.org/licenses/by/ $4.0 /)$.

\begin{abstract}
A comprehensive characterization of various carbon nanotube (CNT) yarns provides insight for producing high-performance CNT yarns as well as a useful guide to select the proper yarn for a specific application. Herein we systematically investigate the correlations between the physical properties of six CNT yarns produced by three spinning methods, and their structures and the properties of the constituent CNTs. The electrical conductivity increases in all yarns regardless of the spinning method as the effective length of the constituent CNTs and the density of the yarns increase. On the other hand, the tensile strength shows a much stronger dependence on the packing density of the yarns than the CNT effective length, indicating the relative importance of the interfacial interaction. The contribution of each physical parameter to the yarn properties are quantitatively analyzed by partial least square regression.
\end{abstract}

Keywords: carbon nanotubes; yarn; electrical conductivity; tensile strength; nanotube length

\section{Introduction}

Carbon nanotubes (CNTs) display very high electrical conductivities, thermal conductivities, as well as mechanical strengths, and sorption abilities [1-5]. Because they also have lower densities than metals such as copper and steel [6], CNTs have potential as alternatingcurrent power cables and wires. In fact, their estimated electrical conductivity is as high as $900,000 \mathrm{~S} / \mathrm{cm}$, which is much larger than that of copper $(600,000 \mathrm{~S} / \mathrm{cm})$ [7]. Additionally, the tensile strength of CNT bundles is $\sim 80 \mathrm{GPa}$, whereas that of steel is $\sim 1 \mathrm{GPa}$ [2].

To preserve superior properties in macroscopic CNT-based structures, the production of yarns composed of CNTs offers a potential for high-strength and lightweight materials that are also thermally and electrically conductive [8-12]. Macroscopic CNT yarns show an electrical conductivity and tensile strength of 10,900 S/ cm and 9.6 GPa, respectively [13-15]. Their values are within a factor of those of aluminum and commercially available carbon fibers, respectively. CNT yarns exhibit a strength similar to carbon fibers and an electrical conductivity similar to metal.

Currently, CNT yarns are mainly produced by three methods: dry spinning from multiwalled CNT (MWCNT) forests, direct spinning from a CVD furnace, and wet-spinning of CNTs (Scheme 1). Elucidating their structures, properties, and relationships are critical to realize practical applications of CNT yarns. 
(b)

Direct dry spinning from CNT furnace

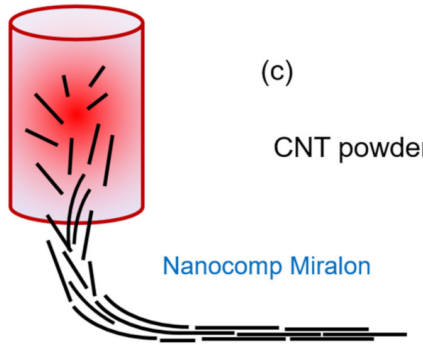

(a) Dry spinning by draw twis process from CNT forest

Taiyo Nippon Sanso Hamamatsu Carbonics

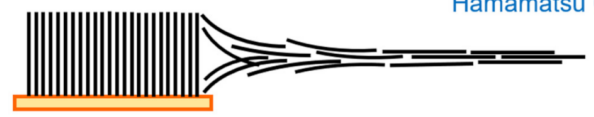

DexMat

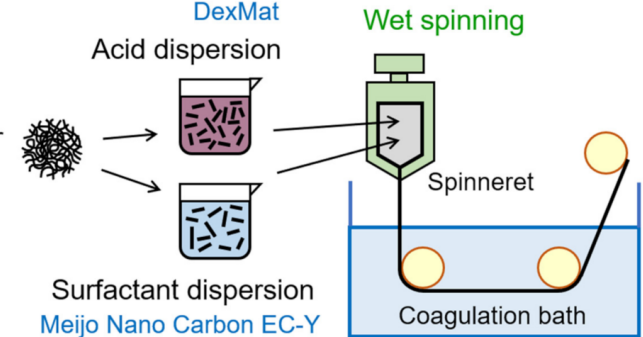

Scheme 1. Schematic representation of spinning methods for CNTs. (a) Dry spinning by draw twist process from CNT forest, (b) dry spinning from CNT furnace, and (c) wet-spinning.

We previously investigated the electrical and mechanical properties of wet-spun CNT yarns using far-infrared (FIR) spectroscopy. These properties are related to the effective CNT length [16-18]. The observed FIR peak can be explained by the one-dimensional plasmon model. Consequently, the estimated length can be ascribed to that of the clean and straight CNT portion between defects or kinks (effective CNT length) [19-22]. FIR spectroscopy is applicable to single-walled CNTs (SWCNTs) and MWCNTs because the resonant frequency is insensitive to the CNT diameter $[19,21]$. On the other hand, the G-band/D-band ratio in the Raman spectrum strongly depends on the CNT diameter and CNT types.

Here, we systematically investigate the structure of various CNT yarns and the properties of the constituent CNTs. Our study evaluated yarns fabricated by three different spinning methods using various CNTs. We observed the yarn structures and cross-sections by scanning electron microscopy (SEM). We also estimated the average CNT diameters and wall numbers by transmission electron microscopy (TEM), the CNT alignment by wide angle X-ray diffraction (WAXD), the G/D ratios and the radial breathing modes by Raman spectroscopy, and the effective CNT lengths by FIR spectroscopy.

Then we evaluated the electrical conductivities and the tensile strengths of the yarns, and their correlation with the yarn and CNT properties. The electrical conductivity of the yarns is significantly correlated with the effective CNT length, yarn density, and CNT alignment in the yarns. In contrast, the tensile strength depends more on the yarn density and the CNT alignment. To quantitatively evaluate the contribution of each physical parameter to the properties of CNT yarn, the measurement data was analyzed using the partial least square (PLS) regression [23-25].

\section{Materials and Methods}

The six commercially available CNT yarns were studied here (Scheme 1). Two CNT yarns by dry spinning from CNT forests were purchased from Hamamatsu Carbonics Corporation (Shizuoka, Japan) and Taiyo Nippon Sanso Corporation (Tokyo, Japan). The CNT yarn by direct spinning from a CVD furnace (Miralon CNT yarn) was obtained from Nanocomp Technologies Inc. (Merrimack, NH, USA). The wet-spinning CNT yarns from surfactant-assisted aqueous dispersion and strong acid dispersion were purchased from Meijo Nano Carbon Co., Ltd. (Aichi, Japan) (EC-Y type I and II) and DexMat Inc. (Houston, TX, USA), respectively.

The morphologies of the CNT yarns were observed by SEM microscopy (Hitachi SU-8200, Tokyo, Japan). The diameter and wall number of the CNTs were estimated by TEM microscopy (EM002B, Topcon, Tokyo, Japan), while WAXD (AichiSR, beam line BL8S3, Aichi, Japan) assessed the CNT alignment in the yarns. The photon energy was $13.48 \mathrm{KeV}(\lambda=0.092 \mathrm{~nm})$. The sample-to-detector distance was $0.208 \mathrm{~m}$. The WAXD 
spectra were measured using a two-dimensional detector (R-AXIS VII++). The scattering wave vector $q=4 \pi \sin \theta / \lambda$, where $\theta$ is the scattering angle. The Raman spectra were measured with inVia (Renishaw, Wotton-under-Edge, England, UK) with an excitation wavelength of $532 \mathrm{~nm}$. The effective length of each CNT was estimated from its FIR optical absorption spectrum [20,22]. The FIR measurements were acquired using Vertex 80v (Bruker Optics, Billerica, MA, USA) and TR-1000 (Otsuka Electronics, Osaka, Japan). The average structural CNT lengths were estimated from the AFM measurements (Shimadzu SFT-4500, Kyoto, Japan).

The electrical resistance of the CNT yarn was measured using the four-terminal method with a probe system (Summit12000, Cascade Microtech, Beaverton, OR, USA) and a semiconductor device analyzer (B1500A, Keysight, Santa Rosa, CA, USA). Three samples were measured for each CNT yarn type. The distance between the two terminals of the differential voltage measurement system was $30 \mathrm{~mm}$. The obtained values were averaged for, at least, three samples for each CNT yarn.

The breaking strengths of the CNT yarns were measured in tensile tests with a micro strain tester (MST-I, Shimadzu, Kyoto, Japan). The test pieces and the test conditions followed the Japanese standard, JIS R 7606, which is a standard protocol for carbon fiber tensile testing. The gauge length was fixed to $25 \mathrm{~mm}$, and the head speed was fixed to $1 \mathrm{~mm} / \mathrm{min}$. Three samples were measured for each CNT yarn type.

Thermogravimetric analysis (TGA) was used to estimate the carbonaceous purity of the CNT yarns with TGAQ500 (TA instruments, New Castle, DE, USA).

PLS models were constructed from the covariance between the standardized response variables (electrical conductivity or tensile strength) and the standardized explanatory variables (physical parameters). Details for select measurement procedures are described in the Supplementary Materials.

\section{Results and Discussion}

\subsection{Structural Characterizations of Commercially Available CNT Yarns and Their Constituent CNTs}

The structural and physical properties of CNT yarn strongly depend on the spinning method [8-12,26]. Figures 1 and 2 show SEM images of the CNT yarns evaluated in this study. Hamamatsu and Taiyo Nippon Sanso yarns were made by dry spinning from CNT forests [27]. Their estimated diameters by laser micrometer are 59 and $31 \mu \mathrm{m}$, respectively (Table 1, see the Supporting Information). This method spins CNTs from a CNT forest by twisting. The twisted structure is visible from the side views (Figure 1). The roundness of the cross-sections exceeds those of the other yarns, reflecting the high controllability of the dry spinning methods (Figure 2a). The network structures in the high-magnification images show the different diameters of the constituent CNTs (Figure S1 in the Supporting Information).

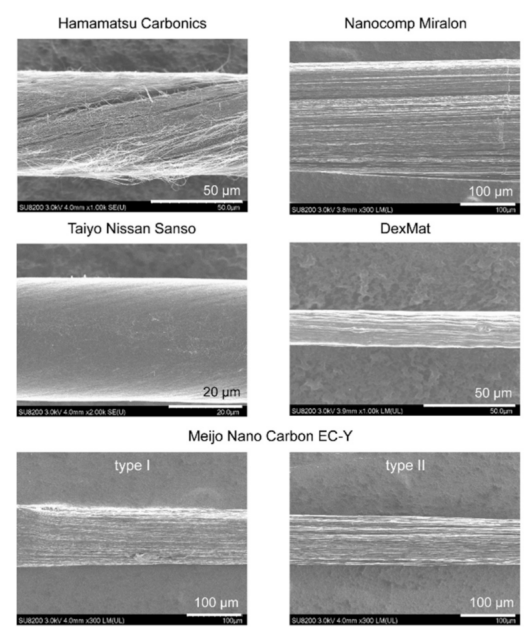

Figure 1. Side-view SEM images of CNT yarns. 
Nanocomp Miralon yarn is produced by direct spinning from a CVD furnace [28]. It has a unique structure among the CNT yarns, including conventional direct spun CNT yarns [29-31]. The low-magnification image shows non-negligible voids in the yarn (Figure 2a), whereas the CNTs seem well packed and sub micrometer voids rarely appear in the high-magnification image (Figure $2 b$ ). The estimated yarn diameter is $230 \mu \mathrm{m}$, which is the largest among the yarns in this study (Table 1).

(a)

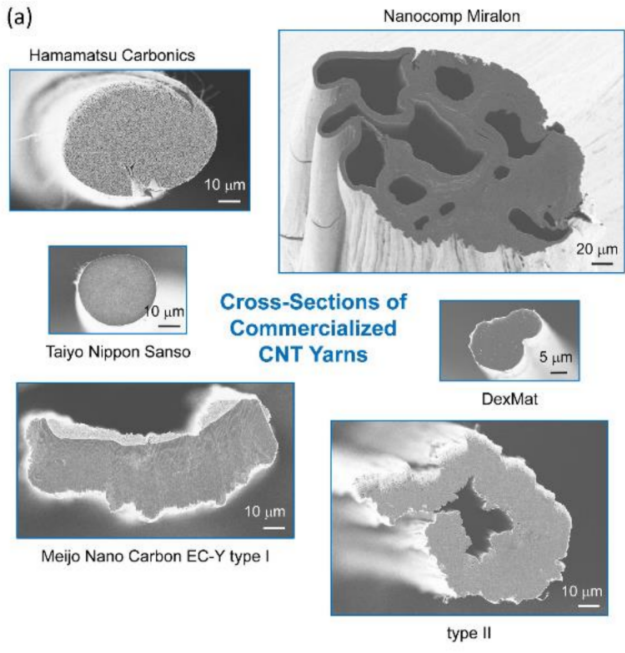

(b)

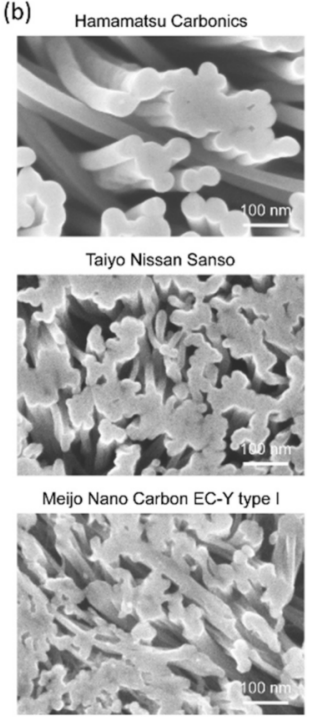

Nanocomp Miralon

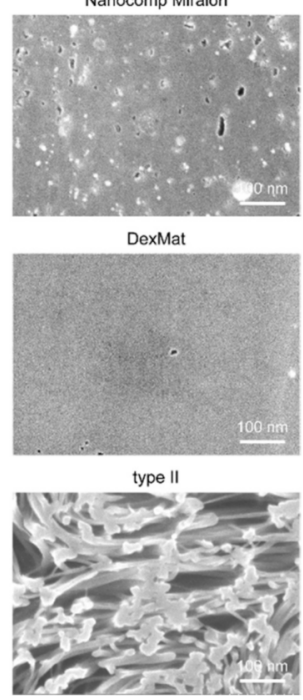

Figure 2. Cross-sectional SEM images of CNT yarns. (a) Low-magnification images. (b) Highmagnification images.

Table 1. Physical properties of the CNT yarns in this study.

\begin{tabular}{|c|c|c|c|c|c|c|}
\hline CNT Yarns & $\begin{array}{c}\text { Hamamatsu } \\
\text { Carbonics }\end{array}$ & $\begin{array}{l}\text { Taiyo Nippon } \\
\text { Sanso }\end{array}$ & $\begin{array}{l}\text { Nanocomp } \\
\text { Miralon }\end{array}$ & $\begin{array}{c}\text { Meijo EC-Y } \\
\text { Type I }\end{array}$ & $\begin{array}{c}\text { Meijo EC-Y } \\
\text { Type II }\end{array}$ & DexMat \\
\hline Yarn diameter $(\mu \mathrm{m})$ & 59 & 31 & 230 & 90 & 76 & 20 \\
\hline Average diameter of CNTs (nm) & $45 \pm 1$ & $12 \pm 0.2$ & $5.3 \pm 0.1$ & $1.8 \pm 0.05$ & $1.7 \pm 0.04$ & $2.2 \pm 0.05$ \\
\hline Average wall number & $50 \pm 2$ & $8.5 \pm 0.1$ & $1.9 \pm 0.03$ & $1.4 \pm 0.05$ & $1.4 \pm 0.05$ & $1.6 \pm 0.05$ \\
\hline CNT type & MWCNT & MWCNT & FWCNT & SWCNT & SWCNT & FWCNT \\
\hline WAXD (002) FWHM (degree) & 42.8 & 23.7 & 9.4 & 21.4 & 38.4 & 7.7 \\
\hline Herman orientation factor & 0.86 & 0.89 & 0.94 & 0.82 & 0.78 & 0.94 \\
\hline G/D ratio & $2.2 \pm 0.03$ & $1.2 \pm 0.03$ & $3.3 \pm 0.3$ & $50 \pm 5$ & $98 \pm 9$ & $31 \pm 2$ \\
\hline CNT effective length (nm) & 270 & 130 & 470 & 2300 & 2300 & 1800 \\
\hline CNT length by AFM $(\mu \mathrm{m})$ & $11 \pm 2^{(a)}$ & $1.1 \pm 0.07^{\text {(a) }}$ & $3.9 \pm 0.4^{(b)}$ & $3.2 \pm 0.2^{(b)}$ & $1.7 \pm 0.1^{(b)}$ & $1.3 \pm 0.1^{(b)}$ \\
\hline Yarn Density $\left(\mathrm{mg} / \mathrm{cm}^{3}\right)$ & 450 & 1111 & 985 & 353 & 575 & 1670 \\
\hline Electrical conductivity $(\mathrm{S} / \mathrm{cm})$ & 462 & 588 & 16,627 & 1725 & 3334 & 70,659 \\
\hline Tensile strength (MPa) & 151 & 716 & 1193 & 69 & 111 & 1595 \\
\hline
\end{tabular}

(a) Individual MWCNTs; (b) Bundles of SW or FWCNTs.

Meijo CNT yarns are wet-spun in a surfactant-assisted aqueous dispersion [32]. These yarns have a ribbon shape, and the CNT bundles are sparsely entangled, rather than aligned (Figure 2). DexMat yarn is produced by wet-spinning CNTs dispersed in a strong acid [10]. DexMat yarn has a rounded cross-section, and CNTs are well packed similar to Nanocomp Miralon (Figure 2). Despite its high density indicated by the cross-section, the side view clearly shows a bundled structure (Figure S1). DexMat has thicker bundles but its average diameter is smaller $(20 \mu \mathrm{m})$ than those of Meijo yarns $(90 \mu \mathrm{m}$ and $76 \mu \mathrm{m}$ for type I and type II, respectively) (Table 1 ). 
The diameters and the wall numbers of the constituent CNTs were estimated from TEM observations (Figure 3 and Figure S2, Table 1). The average values of the CNT diameters are widely distributed from 1.4 to 45 (Figure S2). Based on the observed wall numbers, Hamamatsu Carbonics and Taiyo Nippon Sanso yarns consist of MWCNTs. The other yarns, Nanocomp Miralon, Meijo, and DexMat, contain both FW and SWCNTs.

The CNT alignment along the long axis of the yarns was estimated by WAXD. The WAXD spectra showed a broad peak between $q=11.41$ and $25.27 \mathrm{~nm}^{-1}$, which corresponds to the planes perpendicular to the CNT axis. The CNT alignments were estimated based on the full width at half maximum (FWHM) of the azimuthal scan of the peaks (Figure 4 ). The Hamamatsu and Meijo yarns have Herman orientation factors of 0.78-0.86 (Table 1). On the other hand, DexMat and Nanocomp yarns display higher alignments $(=0.94)$. Note that the broad small peak at an azimuth angle $=150-200^{\circ}$ is X-ray scattering from the Kapton tape, which is used as a window material for the 2D detector and $\mathrm{x}$-ray guideline.

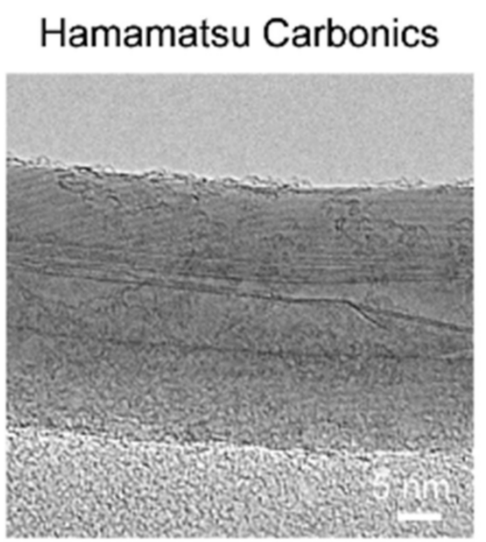

Taiyo Nippon Sanso

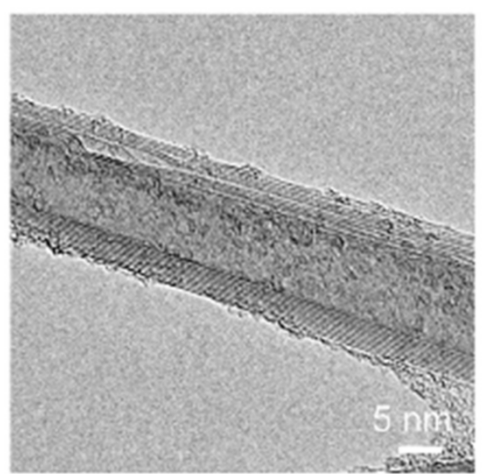

Meijo Nano Carbon EC-Y

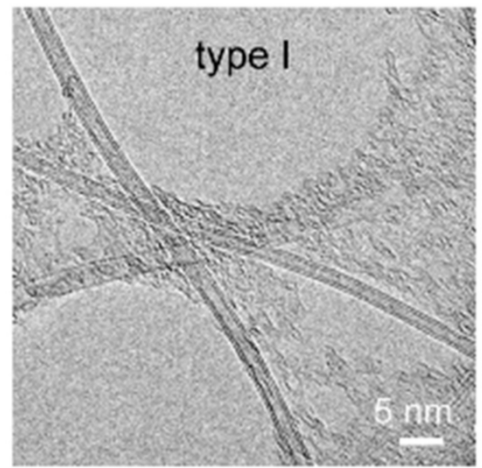

\section{Nanocomp Miralon}

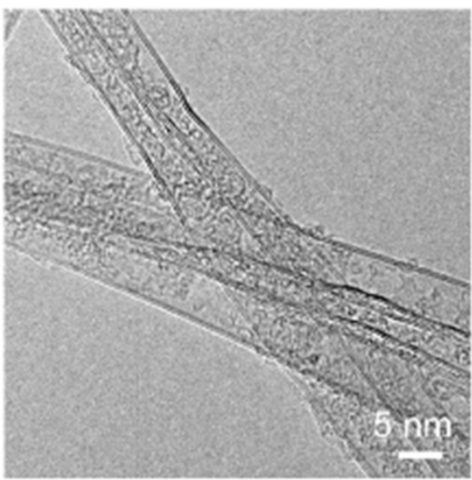

DexMat
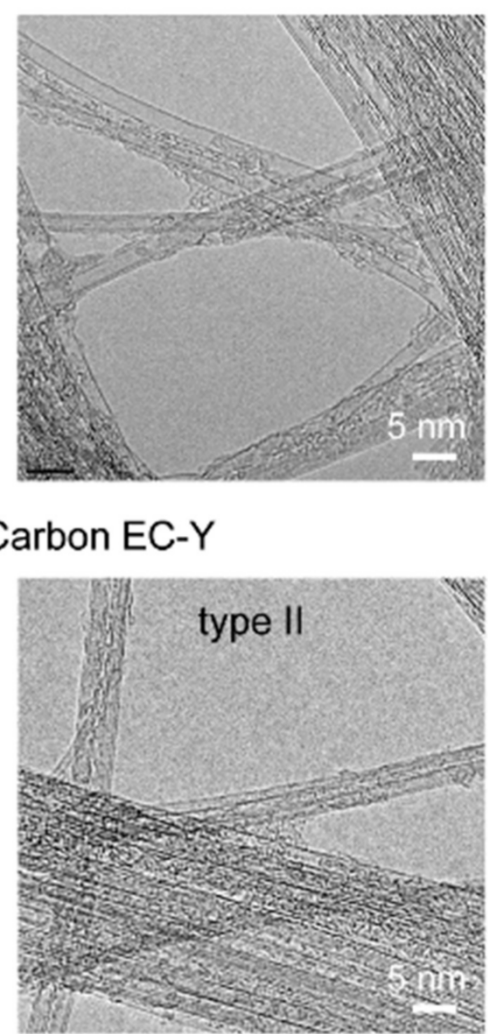

Figure 3. TEM images of constituent CNTs of each yarn. 

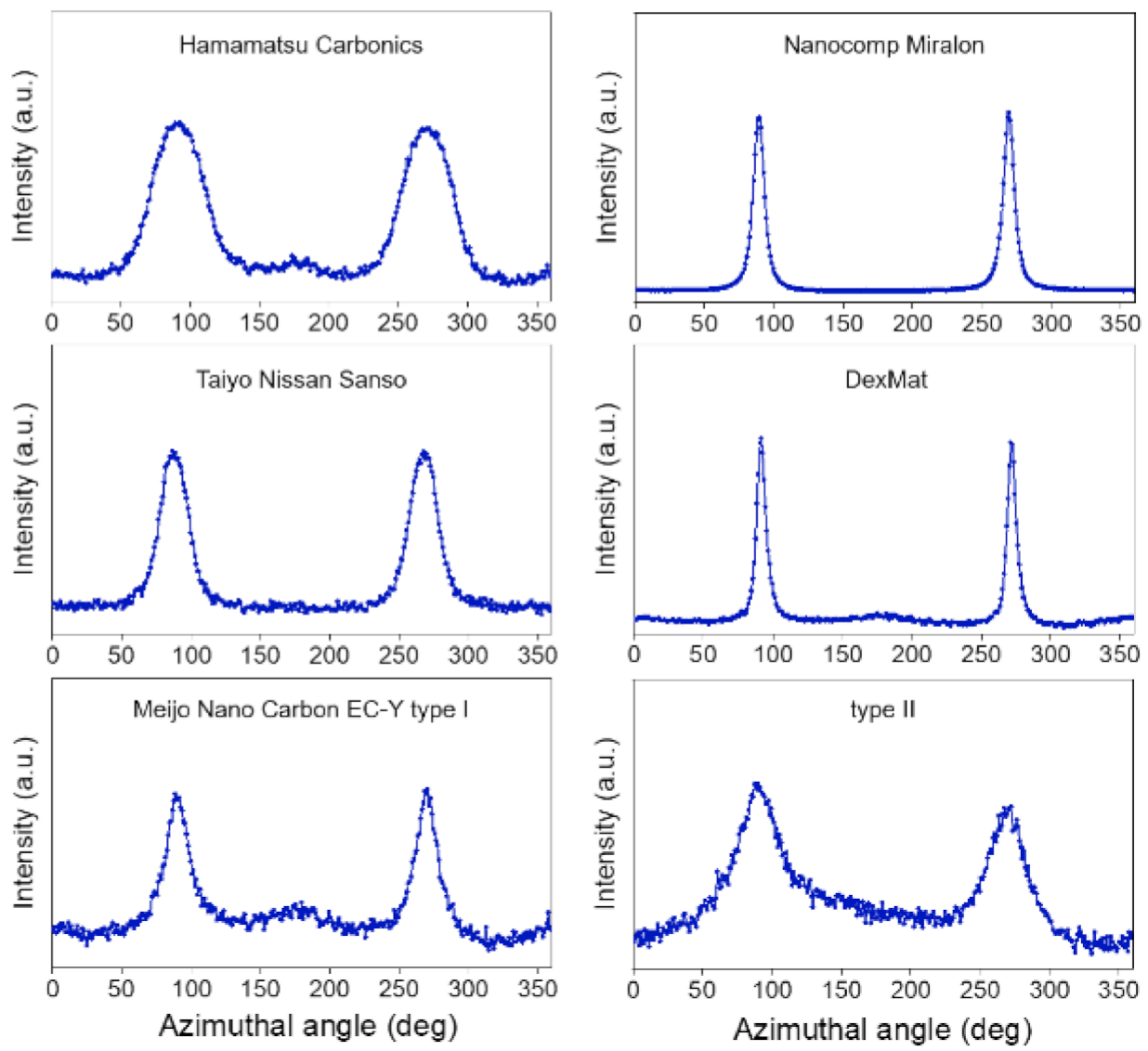

Figure 4. WAXD azimuthal scan of CNT yarns.

\subsection{Spectroscopic Characterizations}

We performed two spectroscopic characterizations (Figure 5). Resonance Raman spectroscopy is a powerful tool for evaluating CNT-based materials (Figure 5a). The integrated G/D intensity ratio of each yarn ranges from 1.2 to 98. Meijo and DexMat tubes show relatively larger G/D values, whereas the Taiyo Nippon Sanso, Hamamatsu Carbonics, and Nanocomp Miralon samples show smaller values around two (Table 1).

Another parameter is the effective length $\left(L_{\text {eff }}\right)$, which is estimated from the optical absorption of CNTs in the FIR region (Figure 5b) [19-22]. Here, the length estimated based on a one-dimensional plasmon resonance model [22] corresponds to the length (or the size) in the high-crystallinity region of the CNT. Therefore, $L_{\text {eff }}$ directly correlates with the physical properties of the CNT films and wet-spun yarns such as electrical conductivity $[16,19]$. SW and FWCNTs have a longer $L_{\text {eff, }}$, whereas MWCNTs show a shorter $L_{\text {eff }}$ (Table 1 ).
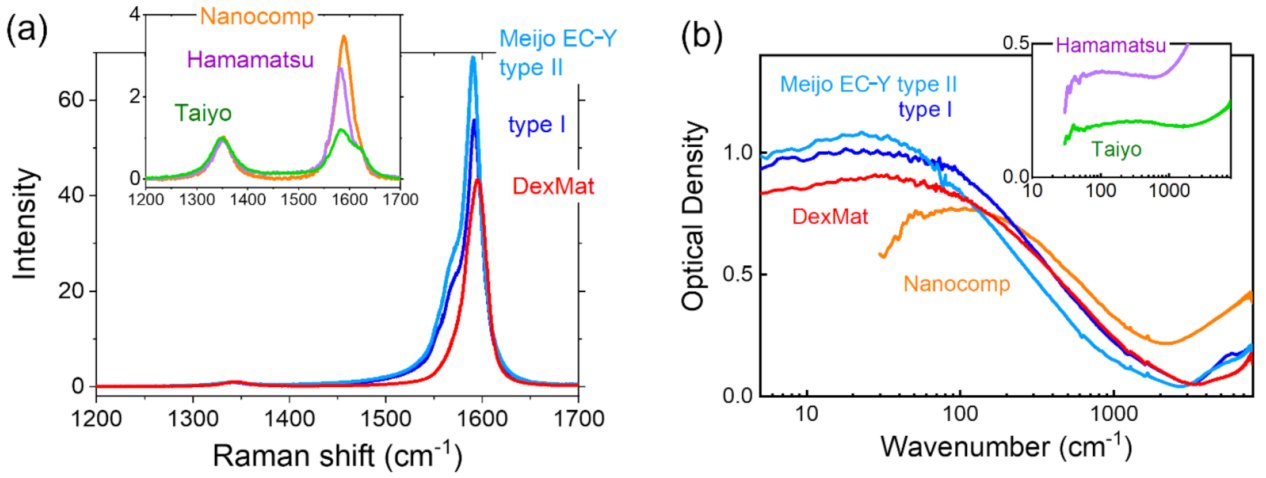

Figure 5. (a) Resonance Raman spectra and (b) far-infrared spectra of the constituent CNTs in yarns. 


\subsection{Electrical Conductivity}

Figure 6a plots the electrical conductivities of the yarns as functions of $L_{\mathrm{eff}}$ of the constituent CNTs. The obtained values are typical for the given spinning method [14,16-18,26,28]. The values range over almost two orders of magnitude. There is no apparent correlation between electrical conductivity and the effective length. However, dividing the CNT yarns into two groups by their density (i.e., high and low densities) reveals a positive dependence on the effective length within the group.

(a)

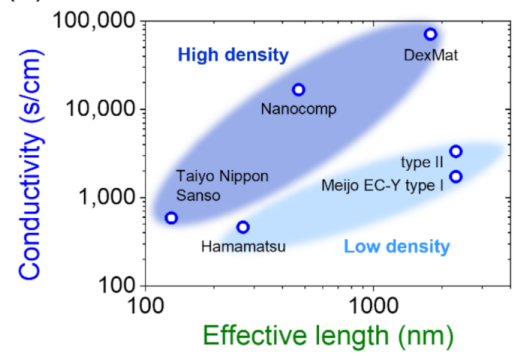

(c)

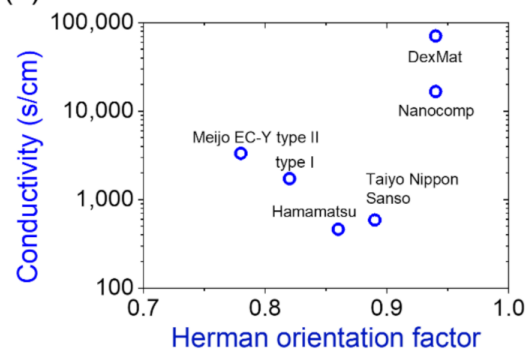

(b)

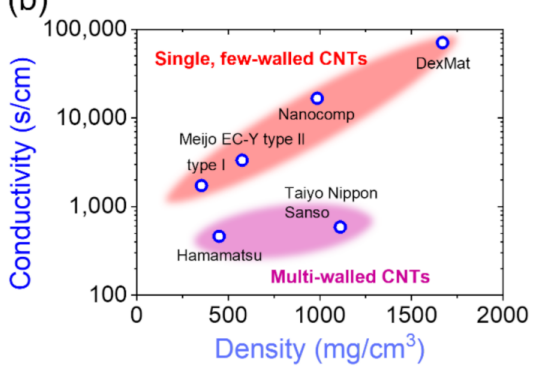

(d)

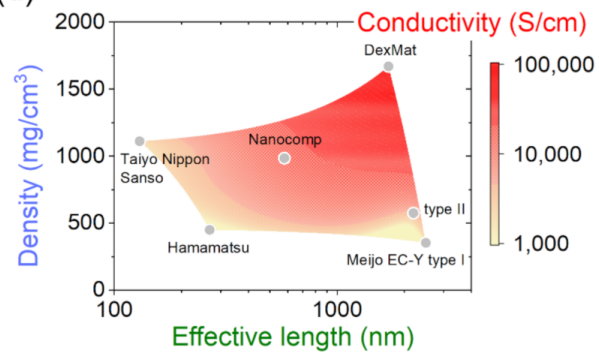

Figure 6. (a) Effective length, (b) density, and (c) Herman orientation factor dependences of the electrical conductivity of yarns. (d) 2D contour plot of the yarn conductivity.

The density dependence of the electrical conductivity does not show a clear trend (Figure $6 \mathrm{~b}$ ). Similar to above, classifying the CNT yarns into two groups depending on the number of walls, (i.e., MWCNTs and FW/SWCNTs) highlights an obvious dependence on the yarn density. Since the wall number of CNTs is usually related to the crystallinity [21], the effective lengths of FW and SWCNTs are longer than those of MWCNTs. Consequently, the electrical conductivity depends on both $L_{\text {eff }}$ (crystallinity) and the yarn density (Figure 6d). If one of these factors is low, the electrical conductivity will be low. In other words, both the CNT effective length and density must be controlled to improve the conductivity of CNT yarn.

The Herman orientation factor dependence of the electrical conductivity (Figure 6c) is similar to the density dependence (Figure $6 \mathrm{~b}$ ). This is reasonable since highly aligned yarns have larger densities (Figures 1 and 2). Indeed, the calculated correlation coefficient between the Herman orientation factors and the yarn densities is 0.78 .

Since $L_{\text {eff }}$ represents the length of the high-crystallinity region of CNTs, CNTs with a longer $L_{\text {eff }}$ should exhibit a higher conductivity because there are fewer CNT junctions per unit length. On the other hand, a common method to estimate the CNT length is performing counting experiments with atomic force microscopy (AFM) $[19,33,34]$. Since the length estimated by the AFM observation corresponds to the physically and structurally connected one, we designated it as the 'structural length' $\left(L_{\text {str }}\right)$ in this paper. As shown in Figure S4a in the Supporting Information, $L_{\text {eff }}$ and $L_{\text {str }}$ do not show a clear correlation, which is consistent with the previous report [19]. Figure S4b shows the electrical conductivity of the yarns as a function of $L_{\text {str }}$ estimated from the AFM observations. The electrical conductivity of Hamamatsu Carbonics yarn is too low for the large $L_{\mathrm{str}}$. This means that although the CNTs are physically connected, defects or kinks on the tube wall cause substantial electrical resistance. 


\subsection{Tensile Strength}

Figure 7a,b show the tensile strengths of CNT yarns as functions of $L_{\text {eff }}$ and the yarn density. The obtained tensile strengths are typical values for CNT yarns produced by the corresponding spinning method [14,16-18,26-28,35,36]. Similar to the conductivity, we classified the results into two groups by the yarn density. Although high-density yarns seem to depend on $L_{\text {eff }}$, low-density yarns do not. This means that $L_{\text {eff }}$ is a good parameter for the tensile strength only if the yarn is sufficiently dense.

Compared with the electrical conductivity, the tensile strength shows a stronger dependence on the yarn density (Figure $7 b$ ) and Herman orientation factor (Figure 7c). Intuitively, CNTs in a dense yarn should have a larger total contact area with neighboring CNTs than those in a low-density yarn. Until the individual CNTs start to slip relative to each other, a greater frictional force is applied to the CNT surface. Thus, dense yarn with a high alignment exhibits a higher tensile strength. Apparently, the increase in friction between CNTs should be higher for longer CNTs. This situation is consistent for CNT yarns with a higher density (Figure 7a).

(a)

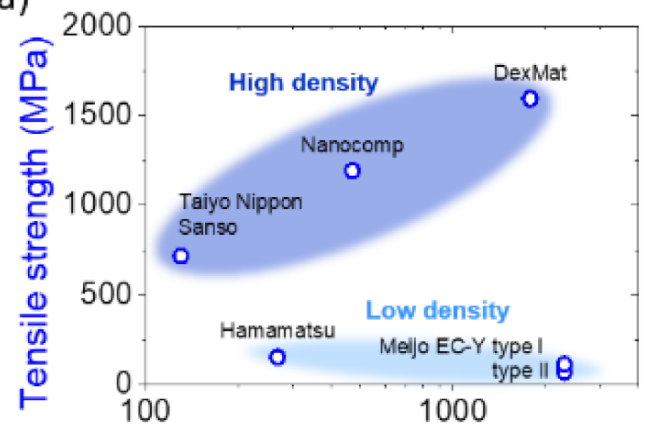

(b)

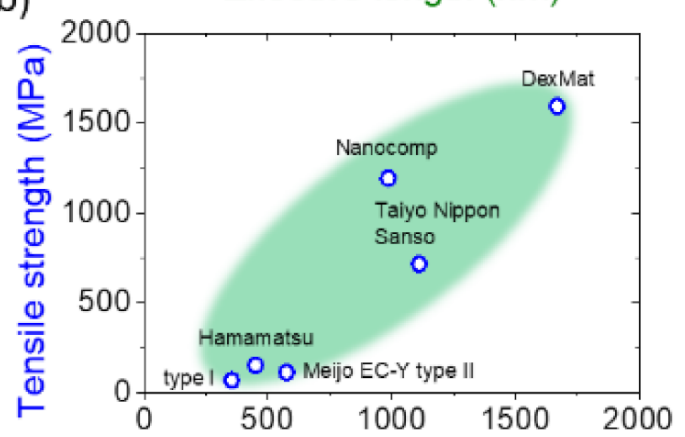

(c)

Density $\left(\mathrm{mg} / \mathrm{cm}^{3}\right)$

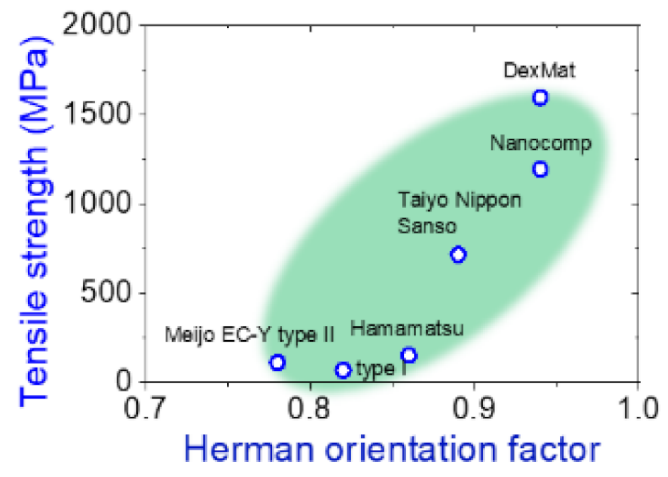

Figure 7. (a) Effective length, (b) density, and (c) Herman orientation factor dependences of the tensile strength of yarns. 


\subsection{PLS Regression Analysis}

The contribution of each physical parameter to the electrical conductivity and the tensile strength was quantitatively analyzed by PLS regression. PLS regression is commonplace in statistics or machine learning, especially for spectroscopic data with high multi-dimensional collinearity [23-25]. PLS regression can efficiently extract information from data even with the multi-dimensional collinearity of variables. Since the absolute values of the regression coefficients of standardized variables $(|\beta|)$ are related to the weights/importance, they are good indicators for interpreting or refining the variables. Based on the results (Figure 8), the contributions of the effective length, yarn density, and degree of orientation are higher than the others for the electrical conductivity. This may indicate that it is important to form a current path with the shortest distance without CNT-CNT junctions in CNT yarns.

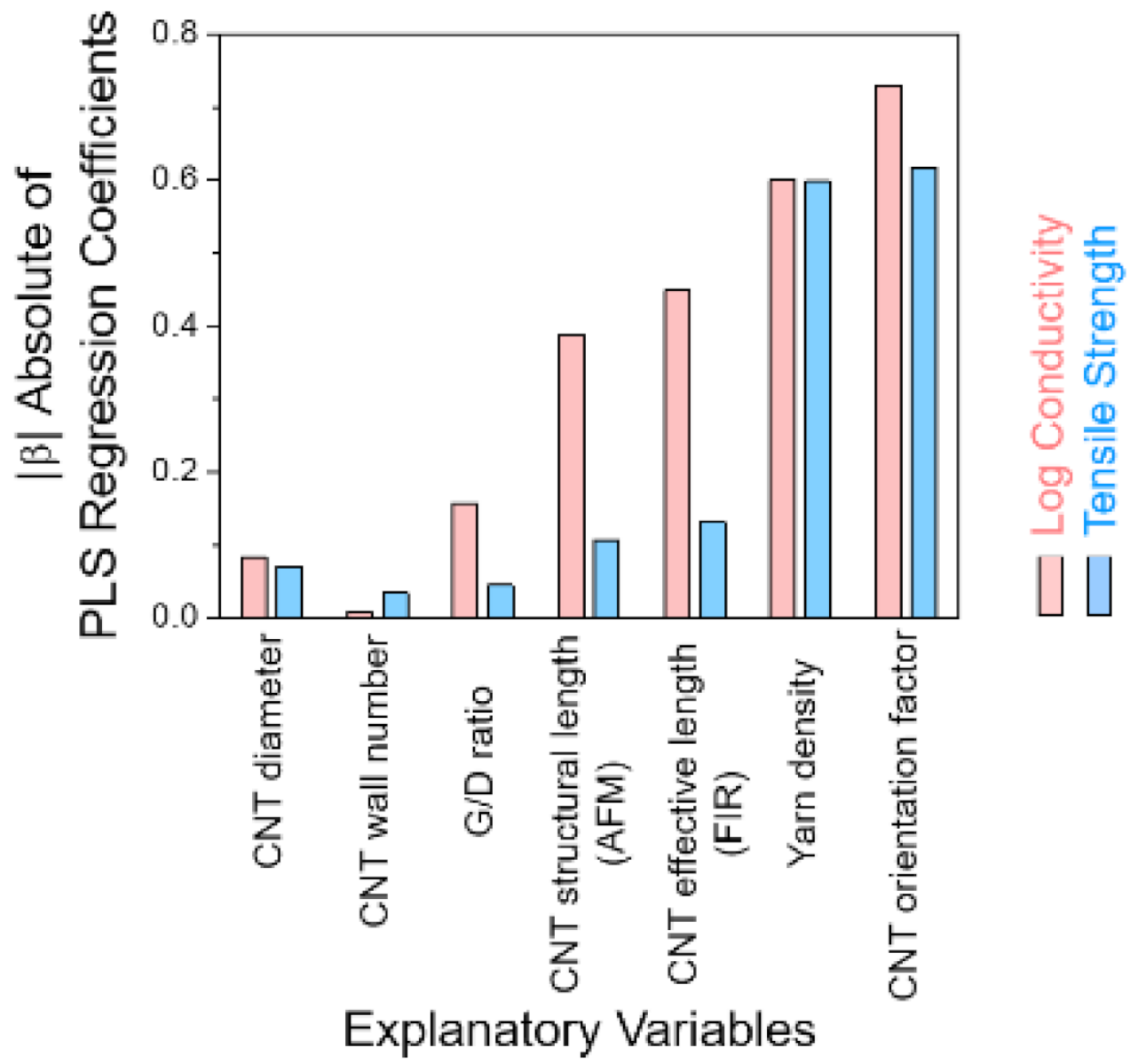

Figure 8. PLS analysis of the electrical conductivity and tensile strength of CNT yarns.

On the other hand, the tensile strength is less dependent on the effective length and the G/D ratio, which are parameters of CNT quality. The density and the Herman orientation factor contribute almost equally. The fracture mechanism of CNT yarn in tension should be dominated by the withdrawal of CNT. Thus, how close a CNT is to other CNTs is more important than the strength of an individual CNT.

\section{Conclusions}

We here conducted comprehensive characterizations of six CNT yarns produced by the different spinning methods with various CNTs. The structural properties of the yarns were investigated by SEM, laser microscope and WAXD. The properties of the constitute CNTs such as diameter, wall number, and effective length were characterized by TEM, resonance Raman, and FIR spectroscopy. The relationship between the physical properties 
of the yarns and the obtained structural parameters were then examined. The CNT effective length and the yarn density well characterize the electrical conductivity of all CNT yarns. On the other hand, the tensile strength of the yarns exhibits much stronger dependence on the yarn density. The stronger correlation of the tensile strength to the yarn density indicates the importance of the interfacial interaction with adjacent CNTs (or CNT bundles) in determining the mechanical properties. PLS analysis can explain the above observations quantitatively. The present study offers the scientific perspectives on the neat CNT yarns. One of the important findings is that DexMat yarn is composed of the longest class of CNTs that we have ever measured [16-20], and the packing density is close to the highest limit [6]. Therefore, to improve the properties of the CNT neat yarn further, post-treatment processes such as doping [10] and cross-linking [37] will become crucial. The doping effects on the electronic structures and the electrical conductivities of DexMat CNT yarn have been investigated by the present research group, which will appear in near future.

Supplementary Materials: The following supporting information can be downloaded at: https:// www.mdpi.com/article/10.3390/nano12040593/s1, Figure S1: Side-view SEM images of CNT yarn at high magnification; Figure S2: (a) TEM images of CNTs used in each yarn. (b) Histograms of CNT diameter and wall number; Figure S3: (a) AFM images of each CNT. (b) Histograms of CNT length; Figure S4: (a) Relationship between structural length and effective length. (b) Structural length dependences of electrical conductivity; Figure S5: (a) Photograph of custom-made fiber diameter measurement system including high-speed optical micrometer (LS-9006MR series, Keyence). (b) Schematic illustration of how to measure yarn diameter. (c) Cross-sectional SEM image of Nanocomp Miralon yarn. (d) Voids extracted from the SEM image by using ImageJ software; Table S1: Carbonaceous content of CNT yarns estimated by TGA.

Author Contributions: T.W., S.Y. (Satoshi Yamazaki), S.Y. (Satoshi Yamashita), and T.I. performed the experiments and analyzed the data. S.M. performed PLS analysis. T.O. and T.W. co-wrote the original manuscript. T.W., S.Y. (Satoshi Yamazaki), S.Y. (Satoshi Yamashita), T.I., S.M., T.M., K.K. and T.O. discussed the results, commented on the manuscript, and approved its submission. All authors have read and agreed to the published version of the manuscript.

Funding: This work was supported by a project (JPNP16010) commissioned by the New Energy and Industrial Technology Development Organization (NEDO).

Institutional Review Board Statement: Not applicable.

Informed Consent Statement: Not applicable.

Data Availability Statement: Not applicable.

Conflicts of Interest: The authors declare no conflict of interest.

\section{References}

1. Li, H.J.; Lu, W.G.; Li, J.J.; Bai, X.D.; Gu, C.Z. Multichannel ballistic transport in multiwall carbon nanotubes. Phys. Rev. Lett. 2005, 95, 080601. [CrossRef] [PubMed]

2. $\quad$ Bai, Y.; Zhang, R.; Ye, X.; Zhu, Z.; Xie, H.; Shen, B.; Cai, D.; Liu, B.; Zhang, C.; Jia, Z.; et al. Carbon nanotube bundles with tensile strength over 80 GPa. Nat. Nanotechnol. 2018, 13, 589-595. [CrossRef] [PubMed]

3. Cornwell, C.F.; Welch, C.R. Very-high-strength (60-GPa) carbon nanotube fiber design based on molecular dynamics simulations. J. Chem. Phys. 2011, 134, 204708. [CrossRef] [PubMed]

4. Zhang, L.; Song, X.; Liu, X.; Yang, L.; Pan, F.; Lv, J. Studies on the removal of tetracycline by multi-walled carbon nanotubes. Chem. Eng. J. 2011, 178, 26-33. [CrossRef]

5. Tabani, H.; Khodaei, K.; Moghaddam, A.Z.; Alexovič, M.; Movahed, S.K.; Zare, F.D.; Dabiri, M. Introduction of graphene-periodic mesoporous silica as a new sorbent for removal: Experiment and simulation. Res. Chem. Intermed. 2019, 45, 1795-1813. [CrossRef]

6. Laurent, C.; Flahaut, E.; Peigney, E.A. The weight and density of carbon nanotubes versus the number of walls and diameter. Carbon 2010, 48, 2994-2996. [CrossRef]

7. Kong, J.; Zhou, C.; Morpurgo, A.; Soh, H.T.; Quate, C.F.; Marcus, C.; Dai, H. Synthesis, integration, and electrical properties of individual single-walled carbon nanotubes. Appl. Phys. A 1999, 69, 305-308. [CrossRef]

8. Koziol, K.; Vilatela, J.; Moisala, A.; Motta, M.; Cunniff, P.; Sennett, M.; Windle, A. High-performance carbon nanotube fiber. Science 2007, 318, 1892-1895. [CrossRef] 
9. Choo, H.; Jung, Y.; Jeong, Y.; Kim, H.C.; Ku, B.-C. Fabrication and Applications of Carbon Nanotube Fibers. Carbon Lett. 2012, 13, 191-204. [CrossRef]

10. Behabtu, N.; Young, C.C.; Tsentalovich, D.E.; Kleinerman, O.; Wang, X.; Ma, A.W.K.; Bengio, E.A.; ter Waarbeek, R.F.; de Jong, J.J.; Hoogerwerf, R.E.; et al. Strong, light, multifunctional fibers of carbon nanotubes with ultrahigh conductivity. Science 2013, 339, 182-186. [CrossRef]

11. Janas, D.; Koziol, K.K. Carbon nanotube fibers and films: Synthesis, applications and perspectives of the direct-spinning method. Nanoscale 2016, 8, 19475-19490. [CrossRef]

12. Yadav, M.D.; Dasgupta, K.; Patwardhan, A.W.; Joshi, J.B. High Performance Fibers from Carbon Nanotubes: Synthesis, Characterization, and Applications in Composites-A Review. Ind. Eng. Chem. Res. 2017, 56, 12407-12437. [CrossRef]

13. Xu, W.; Chen, Y.; Zhan, H.; Wang, J.N. High-strength carbon nanotube film from improving alignment and densification. Nano Lett. 2016, 16, 946-952. [CrossRef] [PubMed]

14. Tsentalovich, D.E.; Headrick, R.J.; Mirri, F.; Hao, J.; Behabtu, N.; Young, C.C.; Pasquali, M. Influence of Carbon Nanotube Characteristics on Macroscopic Fiber Properties. ACS Appl. Mater. Interfaces 2017, 9, 36189-36198. [CrossRef] [PubMed]

15. Taylor, L.W.; Dewey, O.S.; Headrick, R.J.; Komatsu, N.; Peraca, N.M.; Wehmeyer, G.; Kono, J.; Pasquali, M. Improved properties, increased production, and the path to broad adoption of carbon nanotube fibers. Carbon 2021, 171, 689-694. [CrossRef]

16. Wu, X.; Morimoto, T.; Mukai, K.; Asaka, K.; Okazaki, T. Relationship between mechanical and electrical properties of continuous polymer-free carbon nanotube fibers by wet-spinning method and nanotube-length estimated by far-infrared spectroscopy. J. Phys. Chem. C 2016, 120, 20419-20427. [CrossRef]

17. Wu, X.; Mukai, K.; Asaka, K.; Morimoto, T.; Okazaki, T. Effect of surfactants and dispersion methods on properties of single-walled carbon nanotube fibers formed by wet-spinning. Appl. Phys. Express 2017, 10, 055101. [CrossRef]

18. Tajima, N.; Watanabe, T.; Morimoto, T.; Kobashi, K.; Mukai, K.; Asaka, K.; Okazaki, T. Nanotube Length and Density Dependences of Electrical and Mechanical Properties of Carbon Nanotube Fibres Made by Wet Spinning. Carbon 2019, 152, 1-6. [CrossRef]

19. Morimoto, T.; Joung, S.-K.; Saito, T.; Futaba, D.N.; Hata, K.; Okazaki, T. Length-Dependent Plasmon Resonance in Single-Walled Carbon Nanotubes. ACS Nano 2014, 8, 9897-9904. [CrossRef]

20. Morimoto, T.; Ichida, M.; Ikemoto, Y.; Okazaki, T. Temperature dependence of plasmon resonance in single-walled carbon nanotubes. Phys. Rev. B 2016, 93, 195409. [CrossRef]

21. Morimoto, T.; Okazaki, T. Optical resonance in far-infrared spectra of multiwalled carbon nanotubes. Appl. Phys. Express 2015, 8, 055101. [CrossRef]

22. Nakanishi, T.; Ando, T. Optical Response of Finite-Length Carbon Nanotubes. J. Phys. Soc. Jpn. 2009, 78, 114708. [CrossRef]

23. Wold, H. Multivariate Analysis; Academic Press: New York, NY, USA, 1966; p. 391.

24. Muroga, S.; Hikima, Y.; Ohsima, M. Visualization of hydrolysis in polylactide using near-infrared hyperspectral imaging and chemometrics. J. Appl. Polym. Sci. 2018, 135, 45898. [CrossRef]

25. Muroga, S.; Hikima, Y.; Ohsima, M. Near-Infrared Spectroscopic Evaluation of the Water Content of Molded Polylactide under the Effect of Crystallization. Appl. Spectrosc. 2017, 71, 1300-1309. [CrossRef] [PubMed]

26. Headrick, R.J.; Tsentalovich, D.E.; Berdegué, J.; Bengio, E.A.; Liberman, L.; Kleinerman, O.; Lucas, M.S.; Talmon, Y.; Pasquali, M Structure-Property Relations in Carbon Nanotube Fibers by Downscaling Solution Processing. Adv. Mater. 2018, 30, 1704482. [CrossRef]

27. Ghemes, A.; Minami, Y.; Muramatsu, J.; Okada, M.; Mimura, H.; Inoue, Y. Fabrication and mechanical properties of carbon nanotube yarns spun from ultra-long multi-walled carbon nanotube arrays. Carbon 2012, 50, 4579-4587. [CrossRef]

28. Chaffee, J.; Lashmore, D.; Lewis, D.; Mann, J.; Schauer, M.; White, B. Direct Synthesis of CNT Yarns and Sheets. Nsti Nanotech 2008, 3, 118-121.

29. Paukner, C.; Koziol, K.K.K. Ultra-pure single wall carbon nanotube fibres continuously spun without promoter. Sci. Rep. 2014, 4, 3903. [CrossRef]

30. Wang, J.N.; Luo, X.G.; Wu, T.; Chen, Y. High-strength carbon nanotube fibre-like ribbon with high ductility and high electrical conductivity. Nat. Commun. 2014, 5, 3848. [CrossRef]

31. Zhong, X.-H.; Li, Y.-L.; Liu, Y.-K.; Qiao, X.-H.; Feng, Y.; Liang, J.; Jin, J.; Zhu, L.; Hou, F.; Li, J.-Y. Continuous multilayered carbon nanotube yarns. Adv. Mater. 2010, 22, 692-696. [CrossRef]

32. Mukai, K.; Asaka, K.; Wu, X.; Morimoto, T.; Okazaki, T.; Saito, T.; Yumura, M. Wet spinning of continuous polymer-free carbon-nanotube fibers with high electrical conductivity and strength. Appl. Phys. Express 2016, 9, 055101. [CrossRef]

33. Moore, K.E.; Flavel, B.S.; Ellis, A.V.; Shapter, J.G. Comparison of double-walled with single-walled carbon nanotube electrodes by electrochemistry. Carbon 2011, 49, 2639-2647. [CrossRef]

34. Chapkin, W.A.; Wenderott, J.K.; Green, P.F.; Taub, A.I. Length dependence of electrostatically induced carbon nanotube alignment. Carbon 2018, 131, 275-282. [CrossRef]

35. Miao, M. Characteristics of carbon nanotube yarn structure unveiled by acoustic wave propagation. Carbon 2015, 91, 163-170. [CrossRef]

36. Alemán, B.; Reguero, V.; Mas, B.; Vilatela, J.J. Strong Carbon Nanotube Fibers by Drawing Inspiration from Polymer Fiber Spinning. ACS Nano 2015, 9, 7392-7398. [CrossRef]

37. Park, O.K.; Choi, H.; Jeong, H.; Jung, Y.; Yu, J.; Lee, J.K.; Hwang, J.Y.; Kim, S.M.; Jeong, Y.; Park, C.R.; et al. High-modulus and strength carbon nanotube fibers using molecular cross-linking. Carbon 2017, 118, 413-421. [CrossRef] 